\title{
Distributed Kalman filtering using consensus strategies
}

Ruggero Carli
Alessandro Chiuso

\author{
Luca Schenato
}

\author{
Sandro Zampieri
}

\begin{abstract}
In this paper, we consider the problem of estimating the state of a dynamical system from distributed noisy measurements. Each agent constructs a local estimate based on its own measurements and estimates from its neighbors. Estimation is performed via a two stage strategy, the first being a Kalman-like measurement update which does not require communication, and the second being an estimate fusion using a consensus matrix. In particular we study the interaction between the consensus matrix, the number of messages exchanged per sampling time, and the Kalman gain. We prove that optimizing the consensus matrix for fastest convergence and using the centralized optimal gain is not necessarily the optimal strategy if the number of message exchange per sampling time is small. Moreover, we prove that under certain conditions the optimal consensus matrix should be doubly stochastic. We also provide some numerical examples to clarify some of the analytical results.
\end{abstract}

\section{INTRODUCTION}

The recent technological advances in wireless communication and the decreasing in cost and size of electronic devices, are promoting the appearance of large inexpensive interconnected systems, each with computational and sensing capabilities. These complex systems of agents can be used for monitoring very large scale areas with fine resolution. However, collecting measurements from distributed wireless sensors nodes at a single location for on-line data processing may not be feasible due to several reasons among which long packet delay (e.g. due to multi-hop transmission) and/or limited bandwidth of the wireless network, due e.g. to energy consumption requirements.

This problem is apparent in wireless ad-hoc sensor networks where information needs to be multi-hopped from one node to another using closer neighbors. Therefore there is a growing need for in-network data processing tools and algorithms that provide high performance in terms of on-line estimation while (i) reducing the communication load among all sensor nodes, (ii) being very robust to sensor node failures or replacements and packet losses, and (iii) being suitable for distributed control applications.

In this work we will focus on distributed estimation of dynamical systems for which sensor nodes are not physically collocated and can communicate with each other according to some underlying communication network. For example, suppose that we want to estimate the temperature in a building that changes according to a random walk, i.e $T(t+$ $1)=T(t)+w(t)$, where $w(t)$ is a zero-mean random variable with covariance $q$, and we have $N$ sensors that can measure temperature corrupted by some noise, i.e. $y_{i}(t)=$

R. Carli, L. Schenato and S. Zampieri are with the Department of Information Engineering, Università di Padova, Via Gradenigo 6/a, 35131 Padova, Italy. A. Chiuso is with the Dipartimento di Tecnica e Gestione dei Sistemi Industriali, Università di Padova, Stradella S. Nicola, 3 - 36100 Vicenza, Italy, \{carlirug|chiuso|schenato|zampi\}@dei.unipd.it

This work has been supported in part by the national project $\mathrm{New}$ techniques and applications of identification and adaptive control funded by MIUR, and by European Union project SENSNET founded by Marie Curie IRG grant n.014815
$T(t)+n_{i}(t)$, where $n_{i}(t)$ are independent zero-mean random variables with same covariance $r$. If all measurements were instantaneously available to a single location, it is well known from the centralized Kalman filter that the optimal steady state estimator would have the following structure:

$$
\hat{T}(t+1)=\left(1-l^{*}\right) \hat{T}(t)+l^{*} \operatorname{mean}(y(t))
$$

where mean $(y(t)):=\frac{1}{N} \sum_{i=1}^{N} y_{i}(t)$, and $0<l^{*}<1$ is the optimal Kalman gain that depends on the process noise covariance $q$ and the equivalent measurement noise variance $r / N$. This expression already shows two important features of the optimal estimator. The first feature is that the optimal state estimate $\hat{T}(t+1)$ is a weighted average between the previous state estimate $\hat{T}(t)$ and the average of the sensor measurements, thus implying that averaging reduces uncertainty. The second is that the optimal gain needs to be tuned to optimally balance process noise and the equivalent noise of the averaged measurements. In a distributed setting, it is not possible to assume that all measurements are instantaneously available at a specific location, since communication needs to be consistent with the underlying multi-hop communication graph $\mathcal{G}$, and each sensor nodes has its own temperature estimate $\hat{T}_{i}(t)$. However, if it was possible to provide an algorithm that computes the mean of set of number only through local communication, the optimal estimate could be computed at each sensor node as follows:

$$
\begin{aligned}
\hat{T}_{i}(t+1) & =\left(1-l^{*}\right) \operatorname{mean}(\hat{T}(t))+l^{*} \operatorname{mean}(y(t)) \\
& =\operatorname{mean}\left(\left(1-l^{*}\right) \hat{T}(t)+l^{*} y(t)\right)
\end{aligned}
$$

These algorithms are known as average consensus algorithms and can be solved by using updates $z^{+}=Q z$, where $z$ is the vector whose entries are the quantities to be averaged $^{1}$ and $Q$ is a doubly stochastic matrix, i.e. a matrix with properties $Q_{i j} \geq 0, \sum_{j} Q_{i j}=1$ and $\sum_{i} Q_{i j}=1$. Under some weak connectivity properties, these matrices guarantee that $\lim _{m \rightarrow \infty}\left[Q^{m} z\right]_{i}=\operatorname{mean}(z)$, i.e. all elements of vector $Q^{m} z$ converge to their initial mean mean $(z)$. Therefore, provided it is possible to communicate sufficiently fast within two subsequent sensor measurements, i.e. $m \gg 1$, then intuitively we can assume that the following distributed estimation strategy yields the optimal global state estimate:

$$
\begin{array}{rlr}
z & =\left(1-l^{*}\right) \hat{T}_{i}(t)+l^{*} y_{i}(t) \quad \begin{array}{l}
\text { measur. \& } \\
\text { predict. stage }
\end{array} \\
\hat{T}_{i}(t+1) & =\left[Q^{m} z\right]_{i} \quad \text { consensus stage }
\end{array}
$$

Olfati-Saber [6] and Spanos et al. [7] were the first to propose this two-stage strategy based on computing first the mean of the sensor measurements via consensus algorithms, and then to update and predict the local estimates using the centralized Kalman optimal gains. This approach can be extended to multivariable systems where the process evolves according

\footnotetext{
${ }^{1}$ The entries of $z$ can be real numbers, complex numbers or even matrices
} 
to $T(t+1)=A T(t)+w(t)$ and the state is only partially observable, i.e. $y_{i}(t)=C_{i} T(t)+v_{i}$, as shown in the static scenario by Xiao et al. [12] $(A=I, w(t)=0)$ and in the dynamic scenario in [8][5]. In this context, i.e. $m \gg 1$, it natural to optimize $Q$ for fastest convergence rate of $Q^{m}$, which correspond to the second largest singular value of $Q$, for which there are already very efficient optimization tools available [10] [11]. The assumption $m \gg 1$ is reasonable in applications for which communication is inexpensive as compared to sensing. This is the case, for example, in rendezvous control or coordination of mobile sensors where moving and sensing the position is energetically more expensive than transmitting it to their neighbors. However, there are many other important applications in which the number $m$ of messages exchanged per sampling time per node needs to be small, as required in static battery-powered wireless sensor networks. Therefore the assumption that $\left[Q^{m} z\right]_{i} \approx \operatorname{mean}(z)$ is not valid. In this context, for example, it is not clear whether maximizing the rate of convergence of $Q$ is the best strategy. Moreover, also the optimal gain $l$ becomes a function of the matrix $Q$ and the number of exchanged messages $m$, which is unlikely to coincide with the optimal centralized Kalman gain proposed in all the aforementioned papers [6][7][8][5][12].

Recently, Alriksson at al. [1] and Speranzon et al. [9], considered the case $m=1$, i.e. sensors are allowed to communicate only once between sampling instants. In particular, in [1], the authors consider a general MIMO scenario where the matrix $Q=Q(t)$ (=W in their terminology) is selected at each time step in order to minimize the estimation error covariance of each sensor for the next time step, with the only constraint to leave the estimate unbiased corresponding to $\sum_{j} Q_{i j}=1$, i.e. row-sum equal to unity. Also the gain $l=l_{i}(t)$ (=K in their terminology) is different for each sensor and chosen at each time-step using the standard Kalman prediction and correction procedures in order to minimize each sensor estimation error. Simulations show that this iterative algorithm converges and provide good performance, thus providing a methodology to jointly optimize $Q$ and $l$, however the authors do not provide any proof of convergence nor any global optimality guarantee. In fact, this distributed optimization approach greedily minimizes the error covariance of each sensor at each time step, which might not be globally optimal. Differently, in [9] the authors do not separate the algorithm between a consensus stage and an update and correct stage, but they consider a single update equation

$$
\hat{T}(t+1)=K \hat{T}(t)+H y(t)
$$

where $\hat{T}=\left[\hat{T}_{1} \ldots \hat{T}_{N}\right]^{*}$ and $y=\left[y_{1} \ldots y_{n}\right]^{* 2}$, with the additional unbiasedness constraint $\sum_{j}\left(K_{i j}+H_{i j}\right)=1$, i.e. row-sum equal to unity. Using our terminology we note that we would have $K=(1-l) Q$ and $H=l Q$, which satisfy the constraint. Then they propose to compute the design matrices $K, H$ by formulating an optimization problem where at each time step to minimize the sum of all sensor node covariance errors. Similarly to [1], this approach seems to converge and to provide good performance, but once again without any proof of global optimality and insight about the connectivity properties of the underlying graph.

\footnotetext{
${ }^{2}$ The symbol "** denotes the conjugate transpose.
}

In this paper, we want to study the interaction between the consensus matrix $Q$, the number of messages per sampling time $m$, and the gain $l$. With respect with the aforementioned works, we consider a simpler scenario with a scalar state and sensors can measure the state affected by gaussian noise with the same covariance, which still captures some of the most important features of the problem. In fact, also in this simple setup the joint optimization of $Q$ and $l$ is not convex, as discussed in Section V. Our goal is to provide better insights about the problem of distributed estimation using consensus matrices, rather then posing it as a black-box optimization algorithm. Therefore, we explore some important regimes, namely fast communication $m \rightarrow \infty$, "small" measurement noise $(r / q \rightarrow 0)$ and "small" process noise $(q / r \rightarrow 0)$.

This analysis provides useful guidelines for choosing the local filter gain $l$ and the consensus matrix $Q$ also for more general scenarios. As a side result of our analysis we also see that the standard recipe of choosing $Q$ optimizing the second largest eigenvalue is not necessarily the best thing to do; similarly choosing the centralized optimal gain $l_{c}$ is not necessarily the optimal strategy. Moreover, we prove that under certain conditions the optimal matrix $Q$ should be doubly stochastic.

We also provide some numerical examples to clarify some of the analytical results.

\section{Problem formulation}

Consider a set $V$ of $N$ sensor nodes which are labeled $i=$ $1,2, \ldots, N$. These sensors can communicate on a network modeled as a direct graph $\mathcal{G}=(V, E)$, where the edge $(i, j)$ is in $E$ if and only if the node $i$ can transmit its information to the node $j$. We assume that the graph $\mathcal{G}$ is time-invariant. A physical process with state $x \in \mathbb{R}$ evolves according to the continuous-time system

$$
\dot{x}(t)=v(t)
$$

where $v(t)$ is a continuous-time white noise ${ }^{3}$ of zero mean and intensity $q \geq 0$, that is $\mathbb{E}[q(t) q(s)]=q \delta(t-s)$. The initial condition is also a random variable with expectation $x_{0}$ and variance $\sigma$.

Each sensor take measurements of the physical process according to the equation

$$
y_{i}(k T)=x(k T)+n_{i}(k T)
$$

where $T$ is the time-sampling and $k$ the index indicating the $k$-th measure. Note that $y_{i} \in \mathbb{R}, \forall i$. We shall denote $y(k T)=\left[y_{1}(k T), \ldots, y_{N}(k T)\right]^{*}$ and $n(k T)=\left[n_{1}(k T), \ldots, n_{N}(k T)\right]^{*}$. Moreover the noise processes $n_{i}(k T) \in \mathbb{R}$ are such that $\mathbb{E}[n(k T)]=0$, $E[n(k T) n(h T)]=r I \delta_{h k}$ where $\delta_{h k}$ is the Kronecker delta. form

Note also that (2) can be rewritten in the following vector

$$
y(k T)=x(k T) \mathbb{1}+n(k T) .
$$

where $\mathbb{1}=[1 \ldots 1]^{*}$. From now on we assume, without loss of generality, that $T=1$. Suppose now that, between each pair of subsequent measurement update indices $k$ and $k+1$, the each node exchanges $m$ messages; we assume that these transmissions take place at the following times $k+\delta, k+$

\footnotetext{
${ }^{3}$ We recall that what is commonly referred to as "continuous time white noise" can be thought of as the "derivative" of a Wiener process which, unfortunately, is nowhere differentiable. More rigourously $x(t)$ is a Wiener process.
} 
$2 \delta, \ldots, k+(m-1) \delta, k+m \delta$, where $\delta=\frac{1}{m}$. Note that $k+m \delta=k+1$. Moreover suppose that the $\mathrm{i}$ - $t h$ sensor possesses at each of the above indices a estimate of $x(k)$ that, by convention, we indicate by the following notation $\hat{x}_{i}(k+\delta \mid k), \hat{x}_{i}(k+2 \delta \mid k), \ldots, \hat{x}_{i}(k+(m-1) \delta \mid k)$,

$\hat{x}_{i}(k+1 \mid k)$. More compactly we can write

$$
\hat{x}(k+h \delta \mid k)=\left[\hat{x}_{1}(k+h \delta \mid k), \ldots, \hat{x}_{N}(k+h \delta \mid k)\right]^{*} .
$$

We assume that these estimates are updated according to the following rule

$$
\left\{\begin{aligned}
\hat{x}(k \mid k) & =(1-l) \hat{x}(k \mid k-1)+l y(k) \\
\hat{x}(k+h \delta \mid k) & =Q(k) \hat{x}(k+(h-1) \delta \mid k)
\end{aligned}\right.
$$

where $Q(k)$ is a suitable matrix compatible with the communication graph and where $0<l<1, \forall k \geq 0$. From now on we assume that $l(k)=l$ and $Q(k)=\bar{Q}$, i.e. they are constant.

If we impose that $\hat{x}_{i}$ is an unbiased estimator for each $i$ and for each update index, we have that $Q$ must satisfy the following condition

$$
Q \mathbb{1}=\mathbb{1} .
$$

In fact by imposing that $\mathbb{E}[\hat{x}(k+h \delta \mid k)]=x_{0} \mathbb{1}, \forall 0 \leq h \leq$ $m$, it results from the update rule that

$$
\mathbb{E}[\hat{x}(k+(h+1) \delta \mid k)]=\mathbb{E}[Q \hat{x}(k+h \delta \mid k)]=x_{0} Q \mathbb{1}
$$

In order to have that $x_{0} Q \mathbb{1}=x_{0} \mathbb{1}$, for any possible value $x_{0}$, we obtain that (5) must hold. Furthermore if we restrict to nonnegative $Q$, namely a matrix with nonnegative entries, condition (5) imposes that $Q$ is a stochastic matrix. From now on, we assume that $Q$ is stochastic. Moreover the local estimators are initialized by setting $\hat{x}(0 \mid 0)=y(0)$.

Now we define the new variable $\tilde{x}(k+h \delta \mid k)=$ $x(k+h \delta) \mathbb{1}-\hat{x}(k+h \delta \mid k)$ which represents the estimation error. In order to analyze the structure of the recursive equations that $\tilde{x}(k+h \delta \mid k)$ satisfies, it is convenient to rewrite (1) in the following way

$$
x(k+(h+1) \delta)=x(k+h \delta)+w(k+h \delta)
$$

where

$$
w(k+h \delta)=\int_{k+h \delta}^{k+h \delta} v(\tau) d \tau .
$$

Note that $\mathbb{E}[w(k+h \delta)]=0$ and that $\mathbb{E}\left[w^{2}(k+h \delta)\right]=\frac{q}{m}$. By straightforward calculations, we get that, for $h=0$,

$$
\tilde{x}(k \mid k)=(1-l) \tilde{x}(k \mid k-1)-\ln (k)
$$

and, for $1 \leq h \leq m$,

$$
\tilde{x}(k+h \delta \mid k)=Q^{h} \tilde{x}(k \mid k)+\left(\sum_{i=0}^{h-1} w(k+i \delta)\right) \mathbb{1}
$$

In order to analyze the asymptotic properties of the above estimates it is convenient to introduce the following matrices

$$
P(k+h \delta \mid k)=\mathbb{E}\left[\tilde{x}(k+h \delta \mid k) \tilde{x}(k+h \delta \mid k)^{*}\right],
$$

defined for $0 \leq h \leq m$. One can show that $P(k+h \delta \mid k)$ satisfies, for $h=0$,

$$
P(k \mid k)=(1-l)^{2} P(k \mid k-1)+l^{2} r I
$$

and, for $h=m$,

$$
P(k+1 \mid k)=Q^{m} P(k \mid k)\left(Q^{m}\right)^{*}+q \mathbb{1} \mathbb{1}^{*} .
$$

Plugging (11) into (12) and plugging (12) into (11) evaluated at the index $k+1$ we obtain the following recursive equations

$$
\begin{aligned}
P(k+1 \mid k)= & (1-l)^{2} Q^{m} P(k \mid k-1)\left(Q^{*}\right)^{m}+ \\
& +l^{2} r Q^{m}\left(Q^{*}\right)^{m}+q \mathbb{1} \mathbb{1}^{*}
\end{aligned}
$$

and

$$
\begin{aligned}
P(k+1 \mid k+1)= & (1-l)^{2} Q^{m} P(k \mid k)\left(Q^{*}\right)^{m}+ \\
& +(1-l)^{2} q \mathbb{1} \mathbb{1}^{*}+l^{2} r I
\end{aligned}
$$

Since $\hat{x}(0 \mid 0)=y(0)$ we have that $P(0 \mid 0)=r I$ and $P(1 \mid 0)=r Q^{m}\left(Q^{m}\right)^{*}+q \mathbb{1} \mathbb{1}^{*}$. By rewriting the last two recursive equations as expressions depending respectively on $P(1 \mid 0)$ and $P(0 \mid 0)$ we obtain

$$
\begin{aligned}
P(k+1 \mid k)= & (1-l)^{2 k} Q^{k m} P(1 \mid 0)\left(Q^{*}\right)^{k m}+ \\
& +r l^{2} \sum_{i=0}^{k-1}(1-l)^{2 i} Q^{(i+1) m}\left(Q^{*}\right)^{(i+1) m}+ \\
& +q\left(\sum_{i=0}^{k-1}(1-l)^{2 i}\right) \mathbb{1}^{*}
\end{aligned}
$$

and

$$
P(k \mid k)=q \sum_{i=0}^{k-1}(1-l)^{2 i+2} \mathbb{1} \mathbb{1}^{*}+l^{2} r \sum_{i=0}^{k-1}(1-l)^{2 i} Q^{i m}\left(Q^{*}\right)^{i m}
$$

By taking the limit for $k \rightarrow \infty$, we get

$$
\begin{aligned}
\lim _{k \rightarrow \infty} P(k+1 \mid k)= & r l^{2} \sum_{i=0}^{\infty}(1-l)^{2 i} Q^{(i+1) m}\left(Q^{*}\right)^{(i+1) m}+ \\
& +q \frac{1}{1-(1-l)^{2}} \mathbb{1}^{*}
\end{aligned}
$$

and

$\lim _{k \rightarrow \infty} P(k \mid k)=q \frac{(1-l)^{2}}{1-(1-l)^{2}} \mathbb{1}^{*}+r l^{2} \sum_{i=0}^{\infty}(1-l)^{2 i} Q^{i m}\left(Q^{*}\right)^{i m}$

Now let us define the following functionals cost ${ }^{4}$

$$
J_{1}(l, Q ; m, r, q)=\operatorname{tr}\left\{\lim _{k \rightarrow \infty} P(k+1 \mid k)\right\}
$$

and

$$
J_{2}(l, Q ; m, r, q)=\operatorname{tr}\left\{\lim _{k \rightarrow \infty} P(k \mid k)\right\}
$$

We can formulate the following minimization problem.

Problem Given a graph $\mathcal{G}$ and a nonnegative integer $m$, find a real $l$ such that $0<l<1$, and a matrix $Q \in \mathcal{Q}$, minimizing $J_{1}$ or $J_{2}$.

Remark 1: In the sequel of the paper we will consider only $J_{1}$. The reason will be clear in the next sections where the minimization on $J_{1}$ will permit us to retrieve, for some particular cases, the results already known in the literature regarding the Kalman filtering. For the sake of the simplicity,

${ }^{4}$ In the remainder of the paper, when there is no risk of confusion, we might drop some arguments of the cost (e.g. denote $J_{1}(l, Q)$ rather than $\left.J_{1}(l, Q ; m, r, q)\right)$. 
we will denote this functional cost simply by $J$ in place of $J_{1}$. Hence

$$
J=r l^{2} \operatorname{tr}\left\{\sum_{i=0}^{\infty}(1-l)^{2 i} Q^{(i+1) m}\left(Q^{*}\right)^{(i+1) m}\right\}+q \frac{1}{1-(1-l)^{2}} N
$$

Remark 2: Let us denote with $\sigma(Q)=$ $\left\{1, \lambda_{1}, \lambda_{2}, \ldots, \lambda_{N-1}\right\}$ the spectrum of $Q$. Note that, if $Q$ is a normal matrix, namely $Q Q^{*}=Q^{*} Q$ then formula in the previous remark can be rewritten as

$$
J=\frac{r l^{2}+q N}{1-(1-l)^{2}}+r l^{2} \sum_{j=1}^{N-1} \frac{\left|\lambda_{j}\right|^{2 m}}{1-(1-l)^{2}\left|\lambda_{j}\right|^{2 m}}
$$

Also note that if $Q$ is normal and stochastic, then it is also doubly stochastic.

From now on, we will assume that $Q$ is a normal matrix and we will denote by $\mathcal{Q}$ the set of the normal matrices compatible with the graph $\mathcal{G}$. Relevant subclasses of normal matrices are, for instance, Abelian Cayley matrices [4], circulant matrices and symmetric matrices.

\section{OPTIMAL CONSENSUS MATRIX $Q$ FOR FIXED GAIN $l$}

In this section we assume that the estimation gain $l$ is fixed, and thus the problem we want to solve becomes the following

$$
Q(l ; m)=\underset{Q \in \mathcal{Q}}{\arg \min } J(Q, l ; m) .
$$

Although the study of the above problem is quite hard in general, a detailed analysis can be carried out in some interesting situations. In particular in the following we will restrict to the following three cases:

- the communication graph $\mathcal{G}$ is undirected

- the sensors can communicate arbitrarily fast within two subsequent measurements, i.e., $m \rightarrow \infty$

- the estimation gain $l$ is sufficiently large, i.e. $l \rightarrow 1$, which intuitively corresponds to the situation in which the variance of the measurement noise is negligible with respect to the variance of the process, i.e $\frac{r}{q} \approx 0$.

Before proceeding to treat these cases separately, we observe that

$$
\min _{Q \in \mathcal{Q}} J=\frac{r l^{2}+q N}{1-(1-l)^{2}}+\min _{Q \in \mathcal{Q}} r l^{2} \sum_{j=1}^{N-1} \frac{\left|\lambda_{j}\right|^{2 m}}{1-(1-l)^{2}\left|\lambda_{j}\right|^{2 m}}
$$

and hence we can restrict only to the evaluation of last term of the previous equation. Since this quantity will appear often along the section, we denote it as

$$
S(Q, l ; m)=\sum_{j=1}^{N-1} \frac{\left|\lambda_{j}\right|^{2 m}}{1-(1-l)^{2}\left|\lambda_{j}\right|^{2 m}}
$$

\section{A. Undirected communication graph $\mathcal{G}$}

We start by noticing that the assumption that the communication graph $\mathcal{G}$ is undirected implies that, given any $Q \in \mathcal{Q}$, also $Q^{*}$ belongs to $\mathcal{Q}$. Consider now the symmetric matrix $\left(Q+Q^{*}\right) / 2$, that we denote as $Q_{\text {sym }}$. Clearly, $Q_{\text {sym }}$ is normal and it is compatible with $\mathcal{G}$, therefore $Q_{\text {sym }} \in \mathcal{Q}$. The following lemma provides an interesting comparison between $J(Q, l ; m)$ and $J\left(Q_{s y m}, l ; m\right)$ showing that the former is always greater or at most equal to the latter.
Lemma 3.1: Let $Q$ be any matrix in $\mathcal{Q}$ and let $Q_{\text {sym }}$ be defined as above. Then

$$
J(Q, l ; m) \geq J\left(Q_{\text {sym }}, l ; m\right) .
$$

Proof: Let $\lambda_{i}$ be any eigenvalue of $Q$. Then $\operatorname{Re}\left\{\lambda_{i}\right\}$ is an eigenvalue of $Q_{\text {sym }}$, where $\operatorname{Re}\left\{\lambda_{i}\right\}$ denote the real part of $\lambda_{i}$. Clearly $\left|\operatorname{Re}\left\{\lambda_{i}\right\}\right| \leq\left|\lambda_{i}\right|$ which implies that

$$
\frac{\left|\operatorname{Re}\left\{\lambda_{i}\right\}\right|^{2 m}}{1-(1-l)^{2}\left|\operatorname{Re}\left\{\lambda_{i}\right\}\right|^{2 m}} \leq \frac{\left|\lambda_{i}\right|^{2 m}}{1-(1-l)^{2}\left|\lambda_{i}\right|^{2 m}} .
$$

Therefore we have that

$$
S\left(Q_{\text {sym }}, l ; m\right) \leq S(Q, l ; m)
$$

and hence also

$$
J\left(Q_{s y m}, l ; m\right) \leq J(Q, l ; m) .
$$

Remark 3: It is important to note that the previous lemma cannot be generalized to general stochastic matrices $Q$. In fact, it is easy to find a non-normal $Q$ for which the symmetrized matrix $Q_{\text {sym }}$ defined above gives a larger cost index.

An immediate consequence of the above Lemma is that, when the communication graph is undirected, the minimum of the functional cost $J$ is reached by symmetric matrices. Thus, if $\mathcal{Q}_{\text {sym }}$ is the subset of $\mathcal{Q}$ containing the symmetric matrices, that is $\mathcal{Q}_{\text {sym }}=\left\{Q \in \mathcal{Q}: Q=Q^{*}\right\}$, solving (15) is equivalent to solve

$$
\underset{Q \in \mathcal{Q}_{\text {sym }}}{\arg \min } J(Q, l ; m) .
$$

The following result provides a powerful characterization of (15).

Theorem 3.1: Let $\mathcal{Q}_{\text {sym }}$ be as above. Then the functional cost $J(Q, l ; m)$ defined on $\mathcal{Q}_{\text {sym }}$ is a convex function.

Proof: Consider the function $f: \mathcal{B} \subseteq \mathbb{R}^{n} \rightarrow \mathbb{R}$ defined as

$$
f(x)=\sum_{i=1}^{n} \frac{x_{i}^{2 m}}{1-\alpha x_{i}^{2 m}}
$$

where $m \in \mathbb{N}, 0<\alpha<1, x=\left[x_{1}, x_{2}, \ldots, x_{n}\right]^{T}$ and where $\mathcal{B}=\left\{x \in \mathbb{R}^{n}:|x| \leq 1\right\}$. It is easy to verify that the function $f$ is convex and symmetric, i.e. it is invariant to any permutation of the vector entries $x_{i}$. Hence, it follows from the theory of convex spectral functions that also $J$ is a convex function [2].

Theorem 3.1 states that (15) is a convex problem implying thus that the solution of (15) is unique and that it can be performed efficiently by suitable numeric algorithms. In fact, Xiao et al. [11] adopted this strategy to optimize similar performance costs over symmetric stochastic matrices.

\section{B. Fast communication $(m \rightarrow \infty)$}

Before stating the main result of this subsection we recall the following definition. Let $Q$ be any matrix such that $Q \mathbb{1}=$ $\mathbb{1}$ and assume that its spectrum $\sigma(Q)$ is contained in the closed unit disk. Define

$$
\rho(Q)= \begin{cases}1 & \text { if } \operatorname{dim} \operatorname{ker}(Q-I)>1 \\ \max _{\lambda \in \sigma(Q) \backslash\{1\}}|\lambda| & \text { if } \operatorname{dim} \operatorname{ker}(Q-I)=1,\end{cases}
$$


It is called the essential spectral radius of $Q$.

The following result holds.

Theorem 3.2: Let $Q_{1}$ and $Q_{2}$ be two matrices such that $\rho\left(Q_{1}\right)>\rho\left(Q_{2}\right)$. Then there exists $\bar{m}$ (depending only on $\left.\rho\left(Q_{1}\right)-\rho\left(Q_{2}\right)\right)$ such that

$$
\begin{aligned}
& J\left(Q_{1}, l ; m\right) \geq J\left(Q_{2}, l ; m\right), \quad \forall m>\bar{m} . \\
& \text { Proof: } \quad \text { See Appendix }
\end{aligned}
$$

\section{Large gain $(l \rightarrow 1)$}

We start by providing the following notational definition. Given a matrix $A$ we denote with $\|A\|_{F}$ the Frobenius norm of $A$, namely $\|A\|_{F}=\sqrt{\operatorname{tr}\left\{A A^{*}\right\}}$. Given any two matrices $Q_{1}$ and $Q_{2}$ belonging to $\mathcal{Q}$, the following result provides an interesting comparison between $J\left(Q_{1}, l ; m\right)$ and $J\left(Q_{2}, l, m\right)$ when the gain $l$ is sufficiently close to 1 .

Theorem 3.3: Let $Q_{1}, Q_{2}$ be two matrices such that $\left\|Q_{1}^{m}\right\|_{F}>\left\|Q_{2}^{m}\right\|_{F}$. Then there exists $\bar{l}$ (depending only on $\left.\left\|Q_{1}^{m}\right\|_{F}-\left\|Q_{2}^{m}\right\|_{F}\right)$ such that

$$
J\left(Q_{1}, l ; m\right)-J\left(Q_{2}, l ; m\right)>0, \quad \forall l>\bar{l} .
$$

Proof: See Appendix

Remark 4: At first sight, Theorem 3.2 and Theorem 3.3 seem in contradiction. However, this can be explained by observing that $\left\|Q^{m}\right\|_{F}^{2}=1+\rho^{2 m}(Q)+o\left(\rho^{2 m}(Q)\right)$, therefore, for large $m$, minimizing the Frobenius norm of $Q^{m}$ or the spectral radius of $Q$ is almost equivalent.

\section{OPTIMAL GAIN $l$ FOR FIXED CONSENSUS MATRIX $Q$}

In this section we assume that the consensus matrix $Q$ is fixed. Hence the problem we want to solve is the following

$$
\underset{l \in(0,1)}{\arg \min } J(Q, l ; m, r, q)
$$

The previous optimization problem is convex in $l$. This fact can be easily checked by observing that the functional cost $J$ can be written as sum of functions of the form:

$$
g(l)=\frac{x l^{2}}{1-x(1-l)^{2}}, \quad h(l)=\frac{x}{1-x(1-l)^{2}}, x \in[0,1]
$$

which are convex in $l \in(0,1)$.

Consider now a generic matrix $Q \in \mathcal{Q}$ and let

$$
l^{o p t}(Q, m)=\underset{l \in(0,1)}{\arg \min } J(Q, l ; m) .
$$

Convexity of $J$ allows easy computation of $l^{\text {opt }}(Q, m)$.

In the remaining of this section we shall see that the sequence $\left\{l^{\text {opt }}(Q, m)\right\}_{m=0}^{\infty}$ is monotonically non-decreasing in $m$. Moreover, it is bounded below and above by $l_{d}^{o p t}$ and $l_{c}^{\text {opt }}$, which are the optimal gains minimizing $J$ respectively when $Q=I$ and when $Q=\frac{1}{N} \mathbb{1} \mathbb{1}^{*}$, i.e.

$$
l_{d}^{\text {opt }}=\underset{l \in(0,1)}{\arg \min } J(I, l ; m), l_{c}^{\text {opt }}=\underset{l \in(0,1)}{\arg \min } J\left(\frac{1}{N} \mathbb{1}^{*}, l ; m\right)
$$

Note that $Q=I$ and $Q=\frac{1}{N} \mathbb{1} \mathbb{1}^{*}$ represent the two extreme cases in modeling the flow of information between the sensors. Indeed, $Q=I$ corresponds to the situation in which the sensors do not communicate; in such a case there are $N$ Kalman filters running separately (the subscript "d" in $l_{d}^{\text {opt }}$ means decentralized, i.e. no communication). In the other case, instead, we have that the underlying communication graph is complete and this means that each sensor has full knowledge about the estimates of all the other sensors (the subscript "c" in $l_{c}^{o p t}$ means centralized, i.e. full communication). The following proposition characterizes precisely $l_{d}^{o p t}$ and $l_{c}^{\text {opt }}$.

Proposition 4.1: Let $l_{d}^{\text {opt }}$ and $l_{c}^{\text {opt }}$ be as above. Then

$$
l_{d}^{o p t}=\frac{-q+\sqrt{q^{2}+4 q r}}{2 r}, l_{c}^{\text {opt }}=\frac{-q+\sqrt{q^{2}+4 q \bar{r}}}{2 \bar{r}}
$$

where $\bar{r}=\frac{r}{N}$.

The role played by $l_{d}^{o p t}$ and $l_{c}^{o p t}$ is clarified in next proposition where it is shown that they are respectively a lower bound and an upper bound for any $l^{\text {opt }}(Q, m)$. Precisely, it is stated a stronger result characterizing the sequence $\left\{l^{\text {opt }}(Q, m)\right\}_{m=0}^{\infty}$.

Theorem 4.1: Let $Q \stackrel{m=}{\in}$. Let $l^{o p t}(Q, m)$ be defined as above. Then the following chains of inequalities hold true

$$
\begin{aligned}
& l_{d}^{o p t}=l^{o p t}(Q, 0) \leq l^{o p t}(Q, 1) \leq \ldots \leq l^{o p t}(Q, m) \leq \\
& \leq l^{o p t}(Q, m+1) \leq \ldots \leq l^{o p t}(Q, \infty) \leq l_{c}^{o p t}
\end{aligned}
$$

and

$$
\begin{aligned}
& J\left(Q, l_{d}^{o p t} ; 0\right) \geq J\left(Q, l^{o p t}(Q, 1) ; 1\right) \geq \\
& \geq J\left(Q, l^{o p t}(Q, 2) ; 2\right) \geq \ldots \geq J\left(Q, l_{c}^{o p t} ; \infty\right)
\end{aligned}
$$

Moreover $l^{o p t}(Q, \infty)=l_{c}^{o p t}$ if and only if $Q$ is irreducible and aperiodic.

In the interest of space the proof of this theorem and some other theorems in the following sections are omitted and can be found in a longer version of this paper in [3].

\section{JOINT OPTIMIZATION OF $Q$ AND $l$ : SPECIAL CASES}

We have shown in the previous two sections that the functional cost $J$ is a convex function, both if we fix the gain and we assume $J$ defined on the set of the symmetric matrices and if we fix the consensus matrix and we assume that the gain is the independent variable. We ask now whether $J$ is a convex function jointly in $l$ and $Q \in \mathcal{Q}_{\text {sym }}$. The answer is negative, by computing the Hessian of the function $g:[0,1] \times[0,1] \rightarrow \mathbb{R}$ defined as

$$
g(x, y)=\frac{x^{2} y^{2}}{1-(1-x)^{2} y^{2}}
$$

where $x$ and $y$ play the role of $l$ and $\left|\lambda_{j}\right|^{m}$, respectively, in the cost functional $J$. Therefore, the joint minimization of $J$

$$
Q^{o p t}(m, r, q) l^{\text {opt }}(m, r, q) \in \underset{l \in(0,1) ; Q \in \mathcal{Q}}{\arg \min } J(Q, l ; m, r, q)
$$

results to be quite hard in general. Nevertheless, restricting to some asymptotic case on the values of $m, r$ and $q$, it is possible to provide an analytical characterization of the above problem. In particular we will consider the following situations:

- the sensors can communicate arbitrarily fast within two subsequent measurements, i.e., $m \rightarrow \infty$

- $\frac{r}{q} \approx 0$, i.e. the variance of the measurement noise is negligible with respect to the variance of the process

- $\frac{q}{r} \approx 0$, i.e. the variance of the process is negligible with respect to the variance of the measurement noise

First note that $Q^{o p t}(m, r, q) l^{o p t}(m, r, q)$ are indeed only functions of $m$ and $r / q$. In the sequel, without risk of confusion, we shall omit arguments which are kept fixed. 


\section{A. Fast communication $(m \rightarrow \infty)$}

Let $Q^{o p t}(m), l^{o p t}(m)$ be a solution of (19). In this subsection we provide a characterization of $Q^{o p t}(m)$ and $l^{o p t}(m)$ when $m \rightarrow \infty$. Then the following result holds.

Then

Theorem 5.1: Let $Q^{\text {opt }}(\mathrm{m}), l^{\text {opt }}(\mathrm{m})$ be as defined above.

$$
\lim _{m \rightarrow \infty} \rho\left(Q^{o p t}(m)\right)=\min _{Q \in \mathcal{Q}} \rho(Q) .
$$

and

$$
\lim _{m \rightarrow \infty} l^{o p t}(m)=l_{c}^{o p t}
$$

Moreover, if $\arg \min _{Q \in \mathcal{Q}} \rho(Q)$ is a singleton, then also

$$
\lim _{m \rightarrow \infty} Q^{o p t}(m)=\underset{Q \in \mathcal{Q}}{\arg \min } \rho(Q) .
$$

\section{B. Small measurement noise $(r / q \rightarrow 0)$}

In this subsection we treat the case in which the variance of the measurement noise is negligible with respect of the variance of the process, that is $r / q \rightarrow 0$. Let $Q^{o p t}(r / q), l^{o p t}(r / q)$ be a solution of (19), then the following result holds.

Theorem 5.2: Let $Q^{o p t}(r / q), l^{\text {opt }}(r / q)$ be defined above and let

$$
\bar{Q} \in \underset{Q \in \mathcal{Q}}{\arg \min }\left\|Q^{m}\right\|_{F} \text {. }
$$

Then

$$
\lim _{r / q \rightarrow 0}\left\|\left(Q^{o p t}(r / q)\right)^{m}\right\|_{F}=\left\|\bar{Q}^{m}\right\|_{F} .
$$

Moreover

$$
l^{o p t}(r / q)=1-\frac{\left\|\bar{Q}^{m}\right\|_{F}^{2}}{N} \frac{r}{q}+o(r / q) .
$$

In addition if $\arg \min _{Q \in \mathcal{Q}}\left\|Q^{m}\right\|_{F}$ is a singleton also

$$
\lim _{r / q \rightarrow 0} Q^{o p t}(r / q)=\bar{Q} \text {. }
$$

holds.

Note that $l_{c}^{o p t}=1-\frac{1}{N} \frac{r}{q}+o\left(\frac{r}{q}\right)$ and $l_{d}^{o p t}=1-\frac{r}{q}+o\left(\frac{r}{q}\right)$, showing that the communication graph $\mathcal{G}$ determines the coefficient of the first order expansion in $r / q$.

\section{High measurement noise $q / r \rightarrow 0$}

Similarly to the previous section, we now consider the other limiting case for $q / r \approx 0$.

Theorem 5.3: Let $Q^{o p t}(q / r), l^{\text {opt }}(q / r)$ be defined as above and denote with $p(Q)$ the number of eigenvalues of $Q$ on the unit circle. Then

$$
\lim _{q / r \rightarrow 0} p\left(Q^{o p t}(q / r)\right)=\min _{Q \in \mathcal{Q}} p(Q)=: p^{o p t} .
$$

Moreover

$$
l^{o p t}(q / r)=\sqrt{\frac{N}{p^{o p t}}} \sqrt{\frac{q}{r}}+o(\sqrt{q / r}) .
$$

Note that $l_{c}^{o p t}(q / r)=\sqrt{N} \sqrt{q / r}+o(\sqrt{q / r})$ and $l_{d}^{o p t}(q / r)=$ $\sqrt{q / r}+o(\sqrt{q / r})$, therefore, the optimal gain depends on the communication structure of the underlying communication graph. In fact, if sensors cannot communicate, then necessarily $Q^{o p t}=I$, therefore $l^{o p t}(q / r)=l_{d}^{o p t}(q / r)$, while if the communication graph is fully connected, then $Q^{o p t}=\frac{1}{N} \mathbb{1}^{*}$, therefore $l^{o p t}(q / r)=l_{c}^{o p t}(q / r)$.
The previous theorem states also that for $q<<r$ then it is necessary to choose a matrix $Q$ consistent with the communication graph that minimizes the number of unitary eigenvalues.

\section{AN ILlustrative EXAMPLE}

In this section we provide a numerical comparison between the approach presented in this paper and the method proposed in [1]. The authors in [1], analyze a general MIMO scenario where the gain $l=l(t)$ ( $K$ in their terminology) and the consensus matrix $Q=Q(t)$ are time varying quantities which are chosen recursively at each time step. In order to compare the results in [1] with our approach we assume the averaging matrix $W$ in [1] corresponds to performing $m$ consensus iterations using the matrix $Q$, i.e. $W=Q^{m}$. The gain $l$ is chosen to minimize the estimation error covariance of the local estimators (i.e. in a decentralized fashion). In [1] $l$ is different for each sensor; here instead, in order to simplify the analysis, we assume all sensors must have the same gain. The consensus matrix $Q$ is chosen so that the estimation error covariance of the local estimators is minimized after consensus (weighted averaging in [1]).
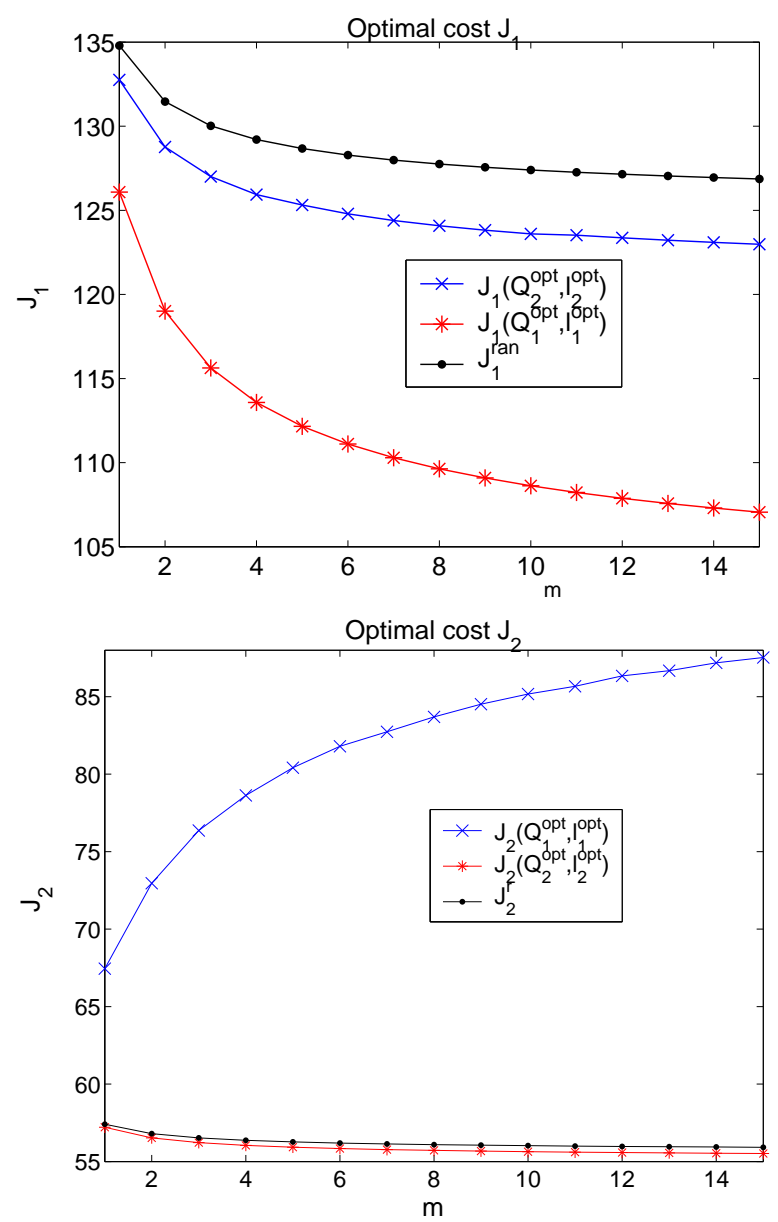

Fig. 1. Comparison between $J_{1}\left(Q_{1}^{\text {opt }}(m), l_{1}^{\text {opt }}(m), m\right)$, $J_{1}\left(Q_{2}^{o p t}(m), l_{2}^{\text {opt }}(m), m\right)$ and $J_{1}^{r}(m)$ (top). Comparison between $J_{2}\left(Q_{2}^{\text {opt }}(m), l_{2}^{\text {opt }}(m), m\right), \quad J_{2}\left(Q_{1}^{\text {opt }}(m), l_{1}^{\text {opt }}(m), m\right) \quad$ and $\quad J_{2}^{r}(m)$ (bottom).

In the simulation reported here, we assume that $N=$ 100, $q=1$ and $r=1$. Moreover we assume that $\mathcal{Q}$ is the 
set of the circulant stochastic matrices of the form

$$
Q=\left[\begin{array}{ccccccc}
1-2 k & k & 0 & 0 & \cdots & 0 & k \\
k & 1-2 k & k & 0 & \cdots & 0 & 0 \\
0 & k & 1-2 k & k & \cdots & 0 & 0 \\
\vdots & \vdots & \vdots & \vdots & \cdots & \vdots & \vdots \\
k & 0 & 0 & 0 & \cdots & k & 1-2 k
\end{array}\right]
$$

where $k$ is a real in $\left[0, \frac{1}{2}\right]$.

We consider both the minimization of $J_{1}$ and $J_{2}$, with $J_{1}, J_{2}$ defined as in the Section II. We use the following notational conventions. $Q_{1}^{o p t}(m), l_{1}^{o p t}(m)$ and $Q_{2}^{o p t}(m), l_{2}^{o p t}(m)$ are the optimal consensus matrices and the optimal gains respectively for $J_{1}$ and $J_{2}$ obtained by solving numerically the problem formulated at the end of Section II, given by:

$$
\begin{aligned}
& Q_{1}^{\text {opt }}(m), l_{1}^{\text {opt }}(m) \in \arg \min _{l \in(0,1), Q \in \mathcal{Q}} J_{1}(Q, l ; m, r, q) \\
& Q_{2}^{\text {opt }}(m), l_{2}^{\text {opt }}(m) \in \arg \min _{l \in(0,1), Q \in \mathcal{Q}} J_{2}(Q, l ; m, r, q)
\end{aligned}
$$

Moreover, $l^{r}(t, m)$ and $Q^{r}(t, m)$ represent the optimal gain and the optimal consensus matrix which are found recursively at the time step $t$ by the method in [1]. The asymptotic values (in $t$ ) of $l^{r}(t, m)$ and $Q^{r}(t, m)$ are denoted respectively by $l^{r}(m)$ and $Q^{r}(m)$ with corresponding asymptotic cost values ${ }^{5} J_{1}^{r}(m):=J_{1}\left(Q^{r}(m), l^{r}(m), m\right)$ and $J_{2}^{r}(m):=J_{2}\left(Q^{r}(m), l^{r}(m), m\right)$. Finally, since the set $\mathcal{Q}$ is parameterized by $k$, we shall identify a matrix $Q$ with the corresponding value of the parameter $k$. Hence, we use $k_{1}^{o p t}(m), k_{2}^{o p t}(m)$ and $k^{r}(m)$ in lieu of $Q_{1}^{o p t}(m), Q_{2}^{o p t}(m)$ and $Q^{r}(m)$.

We run simulations for $m$ ranging in the interval $[1,15]$. A few remarks are now in order. First of all we warn the reader that the iterative (local) optimization proposed in [1] converges to values of the parameters $l^{r}(m)$ and $k^{r}(m)$ which are different from the optimal values obtained minimizing the asymptotic cost, as proposed in this paper (see Figures 2 and 3).

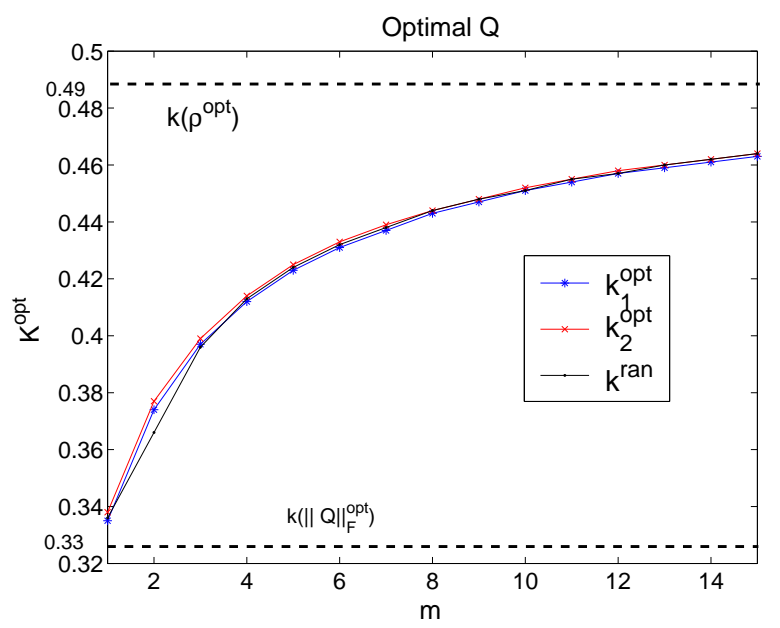

Fig. 2. Optimal consensus matrices $Q$ parameterized by $k$. In the plot the are also reported the values of $k$ which minimize the spectral radius $\rho(Q)$ and the Frobenius norm $\|Q\|_{F}$.

In top panel of Figure 1 we report the value of $J_{1}$ corresponding to the "optimal" parameter pairs

\footnotetext{
${ }^{5}$ There is no proof of convergence in [1]; however this is observed
} experimentally.

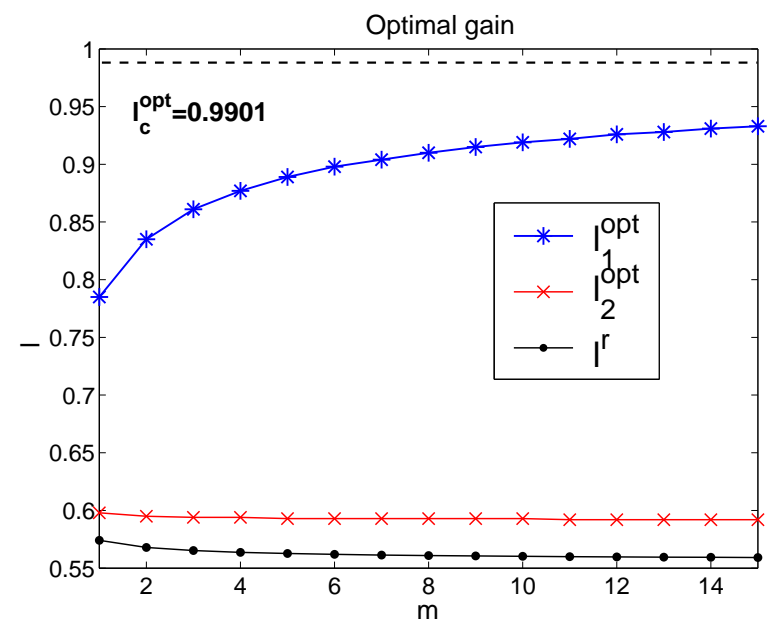

Fig. 3. Optimal gain $l$.

(i) $\quad l_{1}^{o p t}(m), Q_{1}^{o p t}(m), \quad$ (ii) $\quad l_{2}^{o p t}(m), Q_{2}^{o p t}(m)$ and (iii) $l^{r}(m), Q^{r}(m)$. Clearly ${ }^{6} l_{1}^{o p t}(m), Q_{1}^{\text {opt }}(m)$ do better than the others while $l^{r}(m), Q^{r}(m)$ yield the worst performance.

A similar consideration holds for $J_{2}$ (bottom panel of Figure 1. In this case $l_{2}^{o p t}(m), Q_{2}^{o p t}(m)$ give the best performance, only slightly better than $l^{r}(m), Q^{r}(m)$ while $l_{1}^{o p t}(m), Q_{1}^{o p t}(m)$ yield the worst performance. Note in fact that the pair $l_{2}^{o p t}(m), Q_{2}^{o p t}(m)$ is found minimizing $J_{2}$; it is remarkable that, even though $l^{r}(t, m), Q^{r}(t, m)$ minimize step by step an estimation error (and hence a cost which resembles $J_{2}$ ) its asymptotic value does not provide the minimum of $J_{2}$. It is also interesting to observe that $J_{2}\left(l_{1}^{o p t}(m), Q_{1}^{o p t}(m), m\right)$ grows with $m$.

In figure 2 we depict the behavior of $k_{1}^{o p t}(m), k_{2}^{o p t}(m)$ and $k^{r}(m)$, whereas in figure 3 the behavior of $l_{1}^{o p t}(m)$, $l_{2}^{o p t}(m)$ and $l^{r}(m)$.

\section{CONCLUSIONS}

In this paper we have studied a prototypical problem of distributed estimation for Sensor Networks; the state of a scalar linear system is estimated via a two stage procedure which consists in (i) a standard (and decentralized) Kalmanlike update and (ii) information propagation using consensus strategies. To this purpose two design parameters, i.e. the Kalman gain $l$ and the consensus matrix $Q$ have to be designed. This choice is made by optimizing the steady state prediction (or estimation) error. We have discussed, under specific circumstances, the behavior of the "optimal" parameters. This is summarized in table of Figure 4.

Although these results have been obtained for a rather simple scenario where the state is scalar and all sensors are equal, they provide useful guidelines for choosing the local filter gain $l$ and the consensus matrix $Q$ also for more general scenarios. In fact, as discussed in Section $\mathrm{V}$ the joint optimization of $Q$ and $l$ is not convex even in our simple setup.

Finally, we compared our approach with the recursive optimization proposed by Alriksson et al. [1], showing also that their strategy fails to minimize the steady state cost (see Figures 1).

$$
{ }^{6} l_{1}^{o p t}(m), Q_{1}^{o p t}(m) \text { are found minimizing } J_{1} .
$$




\begin{tabular}{|c|c|c|c|}
\hline Section III & undirected graph & $m \rightarrow \infty$ & $l \rightarrow 1$ \\
\hline Fixed $l$ & $\begin{array}{l}Q^{o p t} \text { symmetric } \\
\text { Section III - A }\end{array}$ & $\begin{array}{c}Q^{o p t} \in \underset{Q \in \mathcal{Q}}{\arg \min } \rho(Q) \\
\text { Section III - B }\end{array}$ & $\begin{array}{c}Q^{o p t} \in \underset{Q \in \mathcal{Q}}{\arg \min }\left\|Q^{m}\right\|_{F} \\
\text { Section III }-\mathrm{C}\end{array}$ \\
\hline Section IV & $m=0$ & $0<m<\infty$ & $\begin{array}{c}m \rightarrow \infty \\
+Q \text { aperiodic irreducible }\end{array}$ \\
\hline Fixed $Q$ & $\begin{array}{l}l^{o p t}(Q, 0)=l_{d}^{o p t} \\
\text { Theorem } 4.1\end{array}$ & $\begin{array}{c}l_{d}^{o p t}<l^{o p t}(Q, m) \leq l^{o p t}(Q, m+1)<l_{c}^{o p t} \\
\text { Theorem } 4.1\end{array}$ & $\begin{array}{l}l^{\text {opt }}(Q, \infty)=l_{c}^{o p t} \\
\text { Theorem } 4.1\end{array}$ \\
\hline Section V & $m \rightarrow \infty$ & $r / q \rightarrow 0$ & $r / q \rightarrow \infty$ \\
\hline $\begin{array}{l}\text { Optimal } \\
l \text { and } Q\end{array}$ & $\begin{array}{c}Q^{o p t} \in \underset{Q \in \mathcal{Q}}{\arg \min } \rho(Q), \\
l^{o p t} \rightarrow l_{c}^{o p t} \\
\text { Section } \mathrm{V}-\mathrm{A}\end{array}$ & $\begin{array}{c}Q^{o p t} \in \underset{Q \in \mathcal{Q}}{\arg \min }\left\|Q^{m}\right\|_{F} \\
l^{o p t} \simeq 1-\frac{\left\|\bar{Q}^{m}\right\|_{F}^{2}}{N} \frac{r}{q} \\
\text { Section } \mathrm{V}-\mathrm{B}\end{array}$ & $\begin{array}{c}Q^{o p t} \in \underset{Q \in \mathcal{Q}}{\arg \min } p(Q) \\
l_{c}^{o p t} \simeq \sqrt{\frac{N}{p^{o p t}}} \sqrt{\frac{q}{r}} \\
\text { Section } \mathrm{V}-\mathrm{C}\end{array}$ \\
\hline
\end{tabular}

Fig. 4. Summarizing table of results

\section{APPENDIX}

Proof of Theorem 3.2. Let $\rho_{1}=\rho\left(Q_{1}\right), \rho_{2}=\rho\left(Q_{2}\right)$ and $\epsilon=\rho\left(Q_{1}\right)-\rho\left(Q_{2}\right)$. Observe that

$$
\begin{aligned}
S\left(Q_{1}, l ; m\right) & \geq \frac{\rho_{1}^{2 m}}{1-(1-l)^{2} \rho_{1}^{2 m}} \\
& \geq \frac{\left(\rho_{2}+\epsilon\right)^{2 m}}{1-(1-l)^{2}\left(\rho_{2}+\epsilon\right)^{2 m}} \\
& \geq \frac{\left(\rho_{2}+\epsilon\right)^{2 m}}{1-(1-l)^{2} \rho_{2}^{2 m}}
\end{aligned}
$$

and that

$$
S\left(Q_{2}, l ; m\right) \leq N \frac{\rho_{2}^{2 m}}{1-(1-l)^{2} \rho_{2}^{2 m}} .
$$

Hence, if $\left(\rho_{2}+\epsilon\right)^{2 m} \geq N \rho_{2}^{2 m}$, we have that $S\left(Q_{1}, l ; m\right) \geq S\left(Q_{2}, l ; m\right)$. Straightforward calculations show that this last condition is satisfied if and only if $m \geq \frac{\log N}{2 \log \left(1+\frac{\epsilon}{\rho_{2}}\right)}$. Note that $\frac{\log N}{2 \log (1+\epsilon)} \geq \frac{\log N}{2 \log \left(1+\frac{\epsilon}{\rho_{2}}\right)}$. Therefore by letting $\bar{m}=\left\lceil\frac{\log N}{2 \log (1+\epsilon)}\right\rceil$ the statement of the theorem follows.

Proof of Theorem 3.3. Let $f(l, m)=S\left(Q_{1}, l ; m\right)-$ $S\left(Q_{2}, l ; m\right)$ and $\epsilon=\left\|Q_{1}^{m}\right\|_{F}-\left\|Q_{2}^{m}\right\|_{F}$. Note that $f(l)=\sum_{i=0}^{\infty}\left(\left\|Q_{1}^{(i+1) m}\right\|_{F}^{2}-\left\|Q_{2}^{(i+1) m}\right\|_{F}^{2}\right)(1-l)^{2 i}=$ $\sum_{i=0}^{\infty} \alpha_{i}(1-l)^{2 i}$ where $\alpha_{i}=\left\|Q_{1}^{(i+1) m}\right\|_{F}^{2}-\left\|Q_{2}^{(i+1) m}\right\|_{F}^{2}$. Observe that $\alpha_{0} \geq \epsilon^{2}$ and that, for $i \geq 1, \alpha_{i} \geq-(N-$ 1) $\rho^{2 m(i+1)}$, where $\rho=\rho\left(Q_{2}\right)$. Hence, it follows that

$$
\begin{aligned}
f(l, m) & \geq \epsilon^{2}-(N-1) \sum_{i=1}^{\infty} \rho^{2 m(i+1)}(1-l)^{2 i} \\
& =\epsilon^{2}-\frac{\rho^{4 m}(1-l)^{2}(N-1)}{1-\rho^{2 m}(1-l)^{2}} \geq \epsilon^{2}-\frac{(1-l)^{2}(N-1)}{1-(1-l)^{2}}
\end{aligned}
$$

Clearly, if the last term is nonnegative, then we have that $f(l, m) \geq 0, \forall m$. Straightforward calculations show that this condition is satisfied if and only if

$$
l \geq 1-\sqrt{\frac{\epsilon^{2}}{N-1+\epsilon^{2}}}=\bar{l} .
$$

Therefore, $S\left(Q_{1}, l ; m\right)-S\left(Q_{2}, l ; m\right)>0, \forall l>\bar{l}$ and consequently $J\left(Q_{1}, l ; m\right)>J\left(Q_{2}, l ; m\right)$, thus proving the claim of the theorem.

\section{REFERENCES}

[1] P. Alrickssson and A. Rantzer. Distributed Kalman filtering using weighted averaging. In Proceedings of the 17th Int. Symp. on Math. Theory of Networks and Systems (MTNS'06), Kyoto, Japan, 2006.

[2] J. Borwein and A. Lewis. Convex Analysis and Nonlinear Optimization, Theory and Examples. Canadian Mathematical Society Books in Mathematics. Spring-Verlag, New York, 2000.

[3] R. Carli, A. Chiuso, L. Schenato, and S. Zampieri. Distributed Kalman filtering using consensus strategies. Technical report, Department of Information engineering, University of Padova, 2007. [Online] Available at http://www.dei.unipd.it/ schenato/ PAPERS/KalmanConsensusCDC07.pdf.

[4] R. Carli, F. Fagnani, A. Speranzon, and S. Zampieri. Communication constraints in the state agreement problem. Submitted to Automatica.

[5] R. Olfati-Saber. Distributed Kalman filter with embedded consensus filters. In Proceedings of the 44th IEEE Conference on Decision and Control, and European Control Conference, December 2005.

[6] R. Olfati-Saber and J. Shamma. Consensus filters for sensor networks and distributed sensor fusion. In Proceedings of the 44th IEEE Conference on Decision and Control, and European Control Conference, December 2005.

[7] D. P. Spanos and R. M. Murray. Distributed sensor fusion using dynamic consensus. In Proccedings of the 16th IFAC World Congress, July 2005.

[8] D. P. Spanos, R. Olfati-Saber, and R. M. Murray. Distributed Kalman filtering in sensor networks with quantifiable performance. In Proccedings of the Information Processing for Sensor Networks (IPSN'05), 2005.

[9] A. Speranzon, C. Fischione, and K. Johansson. Distributed and collaborative estimation over wireless sensor networks. In Proceedings of the IEEE Conference on Decision and Control (CDC'06), pages 1025-1030, December 2006.

[10] L. Xiao and S. Boyd. Fast linear iterations for distributed averaging. Systems and Control Letters, 53(1):65-78, September 2004.

[11] L. Xiao, S. Boyd, and S.-J. Kim. Distributed average consensus with least-mean-square deviation. Journal of Parallel and Distributed Computing, 67(1):33-46, September 2007.

[12] L. Xiao, S. Boyd, and S. Lall. A scheme for robust distributed sensor fusion based on average consensus. In Proccedings of the Information Processing for Sensor Networks (IPSN'05), 2005. 